\title{
Phillips 66, debates, artículos docentes, polimedias y visitas de campo: experiencia y valoración por parte del alumnado de grado y máster.
}

\author{
V. Fombuena ${ }^{a}$, L. Quiles-Carrilloa ${ }^{\text {, L. Sanchez-Nacher }}{ }^{a}$, O. Fenollara ${ }^{a}$, D. García- \\ Sanoguera ${ }^{\mathrm{a}}$ \\ a Grupo de Innovación de Prácticas Académicas (GIPA), Universitat Politècnica de València, Plaza \\ Ferrándiz y Carbonell s/n, Alcoy, Alicante (ESPAÑA).
}

\begin{abstract}
The main objective of this communication is to share the experience and appreciation by students of different subjects of degree and master in reference to different teaching methods including activities as diverse as the Phillips 66 method, discussion, use of polymedias and teaching articles and field visits. By means of surveys to the completion of the subjects, the satisfaction and usefulness of each of the new teaching activities applied is valued.
\end{abstract}

Keywords: Phillips 66, Discussion, Polymedia, Teaching Articles, Field Visits.

\begin{abstract}
Resumen
El principal objetivo de la presente comunicación es compartir la experiencia y valoración por parte del alumnado de diferentes asignaturas de grado y de máster en referencia a diferentes métodos docentes entre los que se incluyen actividades tan diversas como el método Phillips 66, debates, uso de polimedias y artículos docentes y visitas de campo. Por medio de unas encuestas a la finalización de las asignaturas se valora la satisfacción y utilidad de cada uno de las nuevas actividades docentes aplicadas.
\end{abstract}

Palabras clave: Phillips 66, Debates, Polimedia, Articulos Docentes, Visitas de Campo.

\section{Introducción}

En el modelo de formación basado en las lecciones magistrales por parte del profesorado, el alumno se muestra como un elemento pasivo y con poca participación (Crispin, 2011). Además el volumen de conocimientos a adquirir en enseñanzas superiores implica la realización de una serie de actividades por parte del alumnado fuera del aula, asumido desde la más pronta edad como una carga extra de trabajo.

Algunos autores como Edgar Dale consideran que si el alumnado participa en metodología activas, el proceso de aprendizaje aumenta, llegando a cuantificarlo en un 50-90\% respecto al 10-30\% de la clase magistral (Edgar, 1969). 
El Grupo de Innovación de Prácticas Académicas (GIPA) considera que es necesaria la innovación continua en la metodología que se aplica en las aulas, pero no solamente son los alumnos los que deben adaptarse y aprender a aprender, sino que el profesorado también requiere de cierta formación y experiencia. Debido a que el profesorado perteneciente al grupo de innovación docente, desarrolla su actividad en el Campus de Alcoy de la Universitat Politècnica de Valencia, se han escogido asignaturas de cuarto curso de Ingeniería Química y asignaturas del Master Universitario de Ingeniería, Procesado y Caracterización de Materiales, por su reducido número de alumnos y por encontrarse en los últimos años de formación universitaria para poner en marcha una serie de actividades relacionadas con las nuevas tecnologías y las clases inversas.

El presente estudio se centra en la evaluación por parte del alumnado de las actividades desarrolladas como alternativas a las tradicionales clases magistrales, pudiendo, de esta forma, poder sacar conclusiones de los puntos fuertes y débiles de cada técnica, sirviendo también para la mejora en la experiencia docente por parte del profesorado.

En el actual trabajo se han evaluado metodologías docentes como el método Phillips 66, la realización de debates sobre aspectos técnicos como partidarios y detractores de la implantación de tecnologías de incineración para el tratamiento de residuos, publicación y uso por parte del alumnado de vídeos polimedia, creación de artículos docentes y por último, las siempre interesantes, prácticas de campo o visitas de instalaciones de empresas relacionadas con los conceptos teóricos vistos en las respectivas asignaturas.

\section{Objetivos}

Desde el curso académico 2017-2018 hasta el presente, el Grupo de Innovación de Prácticas Académicas (GIPA) de la Escuela Politécnica Superior de Alcoy (EPSA) lleva aplicando diferentes actividades de aprendizaje en estudiantes de cuarto curso del Grado de Ingeniería Química (GIQ), y del Master Universitario en Ingeniería, Procesado y Caracterización de Materiales (MUIPCM). Los principales objetivos de dichas actividades se pueden resumir en dos grandes ideas:

- Por un lado, mediante la realización de dichas actividades, los autores tratan de consolidar la información teórica que los estudiantes reciben en el formato tradicional de clase magistral (y en parte substituirla), mediante la realización de diferentes actividades de clases inversas, entre las que cabe destacar el método Phillips 66, la realización de debates sobre una temática en concreto, además del uso de herramientas online como son los vídeos polimedia y los artículos docentes, desarrollados por parte de los profesores involucrados en las asignaturas.

- Por otro lado, se evalúa la opinión de los alumnos que hayan realizado alguna de estas actividades con el fin de dar a conocer su punto de vista sobre esta metodología, novedosa en muchas ocasiones para ellos y de esta forma, con el fin de identificar los puntos fuertes y debilidades de cada técnica para que el propio profesorado pueda incurrir en posibles mejoras en cursos siguientes. 


\section{Desarrollo de la innovación}

3.1. Actividades DesarrolladasEl presente estudio se ha llevado a cabo en las asignaturas de Tratamiento de Residuos Sólidos y Bases de la Ingeniería Química, de segundo y cuarto curso del Grado de Ingeniería Química, así como en las asignaturas optativas de Fabricación de Membranas y sus Aplicaciones y Diagnóstico y Comportamiento en Servicio del Master Universitario de Ingeniería, Procesado y Caracterización de Materiales. La elección de estas asignaturas ha sido debido al reducido número de alumnos, con máximos de 25 por sesión, lo que permite la correcta aplicación de estos métodos, novedosos para la mayoría del profesorado implicado.

A continuación, se detalla una breve explicación de cada una de las actividades desarrolladas:

\subsubsection{Método Phillips 66}

El método Phillips 66 se trata de una técnica de dinámica de grupos aplicada sobre la asignatura de Bases de la Ingeniería Química, asignatura troncal con un máximo de 25 alumnos. El grupo es dividido en subgrupos de unas 6 personas que, durante un máximo de 6 minutos, controlados por el profesor, deberán discutir entre ellos sobre un tema, llegando a una conclusión. Concretamente, en la asignatura, el método Phillips 66 se desarrolló con el objetivo de intercambiar gran cantidad de información con una gestión eficaz del tiempo y sustituir la clase magistral relacionada con la temática de Operaciones Básicas de la Ingeniería. Se ha efectuado una única actividad de esta metodología en la asignatura en una de las clases de 2 horas de duración.

Cada uno de los subgrupos elige un portavoz y se le asigna una serie de operaciones básicas de la ingeniería química, que tras seis minutos de búsqueda de información y discusión, deberán explicar al resto de compañeros. Para la búsqueda de información, el profesorado permitirá el uso de móviles por parte del alumnado.

Del informe de todos los subgrupos se realizará una tabla resumen durante unos 40 minutos donde se muestre de forma esquemática las principales características de cada una de las operaciones básicas de la ingeniería como pueden ser la adsorción, absorción, trituración, extracción líquido-líquido, gasificación, etc.

\subsubsection{Debates}

Uno de los temas de la asignatura de cuarto curso de Tratamiento de Residuos Sólidos es la aplicación de métodos térmicos para la revalorización de residuos, como es el caso de las incineradoras. En clases previas el profesorado ha explicado la tecnología de incineración desde un punto de vista técnico, medioambiental, social y económico. Con el el objetivo de afianzar estos conocimientos y con la ayuda de diferente material aportado por parte del profesor: artículos científicos, noticias de periódicos, informes de Greenpeace, se llevará a cabo un debate sobre partidarios y detractores del uso de incineradoras de residuos sólidos para la correcta gestión de los residuos sólidos urbanos.

El grupo de unos 10 alumnos, puesto que se trata de una optativa de cuarto curso, es dividido en dos grupos. Se les otorga un total de 30 minutos para que busquen argumentos que defiendan su postura, teniendo en cuenta los aspectos vistos en las clases magistrales y los 
Phillips 66, debates, artículos docentes, polimedias y visitas de campo: experiencia y valoración por parte del alumnado de grado y máster.

nuevos datos aportados por los artículos, noticias, etc. Tras este periodo de tiempo, el portavoz de cada uno de los grupos inicia una breve explicación de 3 minutos con los principales argumentos. A partir de este momento, el profesor, en la figura de moderador, irá planteando cuestiones técnicas, sociales y económicas con el fin de asegurarse tratar todos los aspectos esenciales.

Tras la finalización del debate tras 60 minutos se realizará en la pizarra un resumen de los principales aspectos tratados. En la presente asignatura únicamente se ha llevado a cabo un debate en el curso académico 2018-2019.

\subsubsection{Videos polimedias y artículos docentes}

Polimedia es un sistema diseñado por la UPV para la creación de contenidos multimedia como apoyo a la docencia presencial, que abarca desde la preparación de material docente hasta la distribución a través de medios tan atractivos para el alumno como Youtube.

Durante los últimos cursos académicos, el profesorado de la asignatura de Diagnóstico y Comportamiento en Servicio del Master Universitario de Ingeniería, Procesado y Caracterización de Materiales ha desarrollado diferentes videos polimedia con una duración máxima de 10-12 minutos donde se explican los principales conceptos teóricos y prácticos de la asignatura. El principal objetivo es la disponibilidad por parte del alumno de este elemento atractivo de aprendizaje así como servir de apoyo para los conceptos que por falta de disponibilidad horaria no han podido desarrollarse en clase (Zabalza, 2016). De la misma forma, se pone a disposición del alumnado, diferentes artículos docentes con el planteamiento y resolución de breves problemas. Dado que se trata de una metodología donde el alumno es más autónomo se han puesto a disposición 2 artículos docentes y 9 polimedias como apoyo al presente curso académico. Un ejemplo de los mismos se puede observar en las Figuras 1 y 2 .

La utilidad que percibe el alumnado de este tipo de herramientas, así como el uso que le dan será evaluado por parte de encuestas anónimas a la finalización de la asignatura, con el objetivo de recabar información y posibles mejoras.

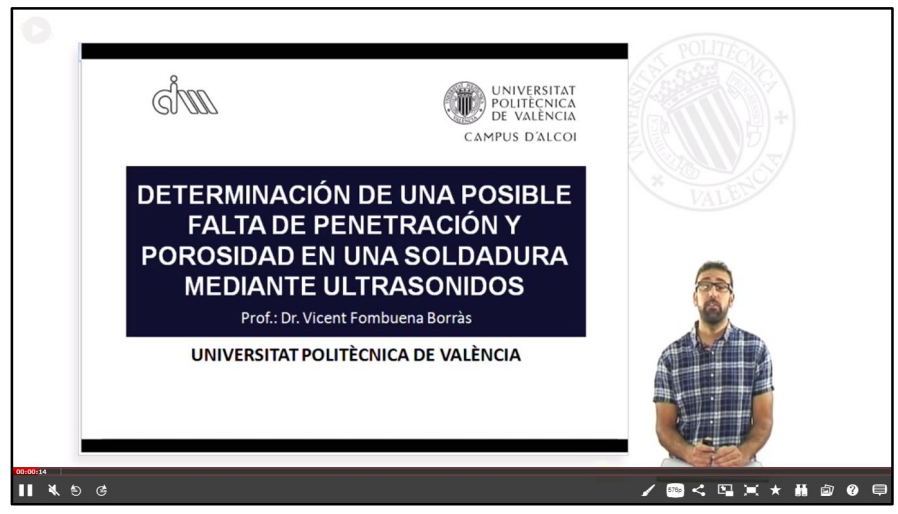

Fig. 1 Ejemplo de polimedia usado en la asignatura 


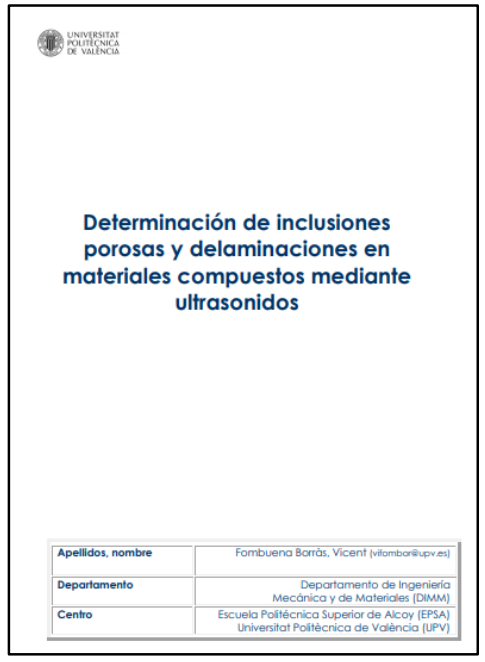

Fig. 2 Ejemplo de artículo docente usado en la asignatura

\subsubsection{Visitas de Campo}

Dentro de la asignatura de Tratamiento de Residuos Sólidos del Grado de Ingeniería Química se realizan una serie de visitas o viajes de campo, a diferentes empresas relacionadas con el tratamiento de residuos, como es el caso de la planta de selección de envases ligeros de Vaersa en Picassent, Valencia (Figura 3). Dada la limitación horaria de este tipo de actividades únicamente se realiza una visita de campo durante la asignatura de Tratamiento de Residuos.

El profesorado de la asignatura considera, que dentro de las enseñanzas técnicas, los viajes de campo son una herramienta útil para superar las limitaciones asociadas a la enseñanza del aula, así como para promover la asimilación de experiencias por parte del alumnado. La visita de campo tiene en todo momento un enfoque docente, donde sobre las mimas instalaciones, el profesor va recordando aquellos conceptos teóricos vistos en clase. Además, en este tipo de instalaciones, reciben la charla y explicación del jefe de planta, cuya formación está muchas veces relacionado con sus estudios, con lo que se ofrece al alumnado un ejemplo sobre posibles salidas laborales.

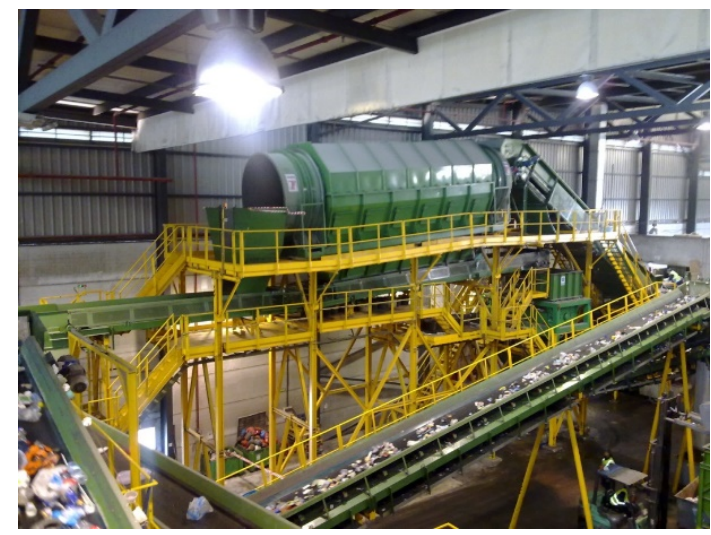


Phillips 66, debates, artículos docentes, polimedias y visitas de campo: experiencia y valoración por parte del alumnado de grado y máster.

Fig. 3 Planta de selección de envases ligeros de Picassent visitada en las prácticas de campo

La utilidad, sensaciones y opiniones por parte del alumnado de este tipo de actividades que apoya la clase magistral y sustituye a las prácticas de laboratorio es obtenida por medio de encuestas anónimas a la finalización de la asignatura.

\subsection{Encuestas de evaluación}

La experiencia por parte del profesorado ha sido satisfactoria. Las actividades desarrolladas permiten alternativas al tradicional uso de presentación y clase magistral. Aunque algunas actividades suponen un mayor tiempo de preparación previa, la experiencia positiva en el aula se traduce en su posterior implantación en próximos años académicos.

Para conocer la opinión con las metodologías propuestas de los alumnos de grado y máster, se ha desarrollado una encuesta básica idéntica para todas las asignaturas piloto. Dado que se trata de una experiencia exploratoria y de búsqueda de percepciones personales, la Figura 4 muestra la encuesta pasada:

\begin{tabular}{|c|c|}
\hline \multicolumn{2}{|r|}{ Grupo de Innovación de Prácticas Académicas (GIPA), } \\
\hline ENCUI & STA SATISFACCIÓN ACTIVIDADES DE DOCENCIA INVERSA \\
\hline $\begin{array}{l}\text { 1. } \mathrm{M} \\
\square \\
\square \\
\square \\
\square \\
\square\end{array}$ & $\begin{array}{l}\text { rque con un X la actividad a evaluar: } \\
\text { Método Phillips } 66 \\
\text { Debate } \\
\text { Vídeo Polimedia } \\
\text { Artículo Docente } \\
\text { Visita de Campo }\end{array}$ \\
\hline 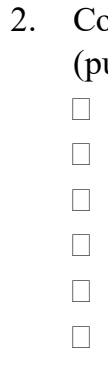 & $\begin{array}{l}\text { no consideras el método aplicado comparado con una clase magistral } \\
\text { edes marcar más de una opción): } \\
\text { Dinámico } \\
\text { Fácil de aprender nuevos conocimientos } \\
\text { Permite la mejora del trabajo en grupo } \\
\text { Permite mejorar la exposición oral de ideas } \\
\text { Entretenido, pero poco útil } \\
\text { De muy poca utilidad }\end{array}$ \\
\hline $\begin{array}{l}\text { Qu } \\
\text { op } \\
\square \\
\square \\
\square \\
\square \\
\square\end{array}$ & $\begin{array}{l}\text { desventajas principales le encuentras al método (puedes marcar más de una } \\
\text { ión): } \\
\text { Ninguna } \\
\text { No se tiene suficiente tiempo para la actividad } \\
\text { No se tienen los conocimientos necesarios para trabajar esta metodología } \\
\text { El resultado depende mucho del grupo de trabajo } \\
\text { No se obtiene una conclusión al trabajo realizado }\end{array}$ \\
\hline
\end{tabular}


4. Mejorarías alguna cosa del método (puedes marcar más de una opción):

$\square \quad$ Ninguna

$\square \quad$ El tiempo para la actividad

$\square \quad$ Ejemplos previos

$\square \quad$ Mayor formación previa

$\square$ Solo es aplicable a algunas asignaturas

5. Del 1 al 10, siendo 1 muy mala y 10 muy buena, marca el rango de satisfacción con la actividad:
$8-10$
$6-8$
4-6
$2-4$

$\square \quad 0-2$

Fig. 4 Encuesta facilitada al alumnado

\section{Resultados}

Las siguientes gráficas (Figuras 5 a 8 ) muestran los resultados obtenidos. Dado que en algunas cuestiones es posible marcar más de una opción, el valor muestra el porcentaje de alumnos encuestados que han marcado esa opción:

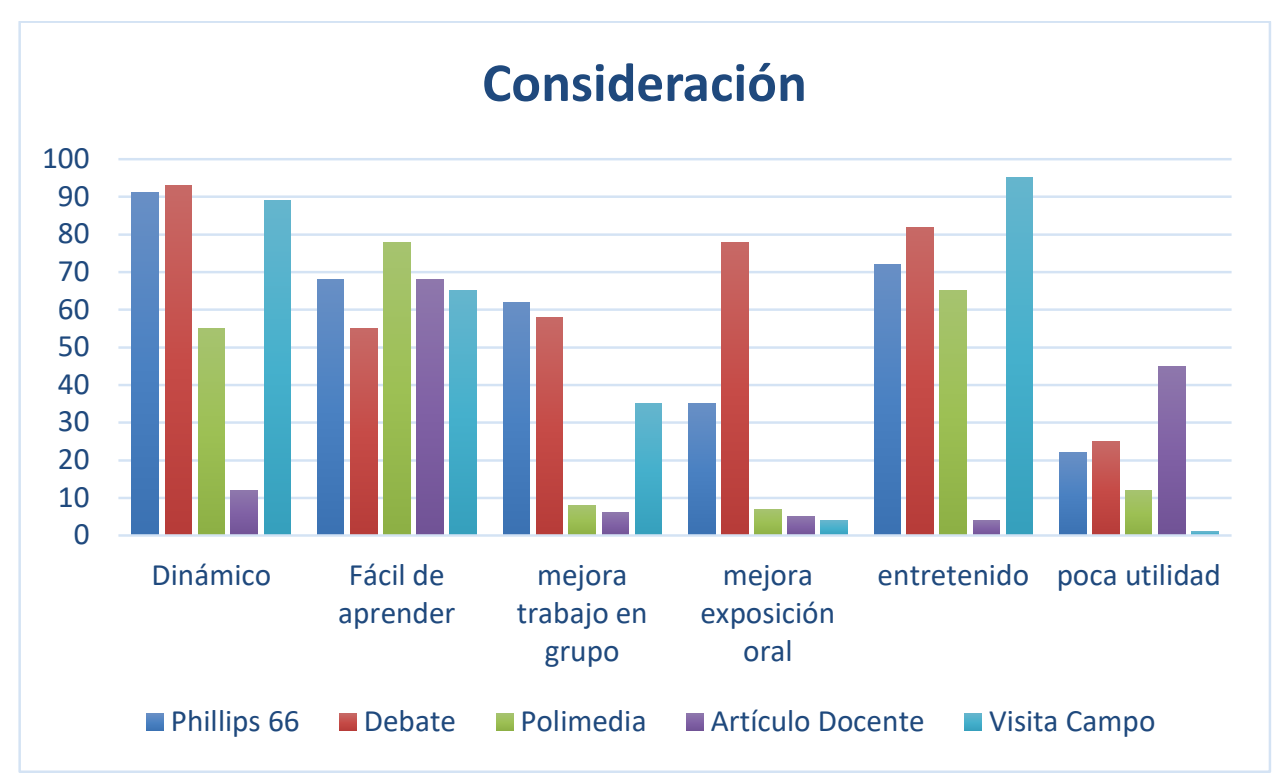

Fig. 5 Consideración por parte del alumnado de la actividad 
Phillips 66, debates, artículos docentes, polimedias y visitas de campo: experiencia y valoración por parte del alumnado de grado y máster.

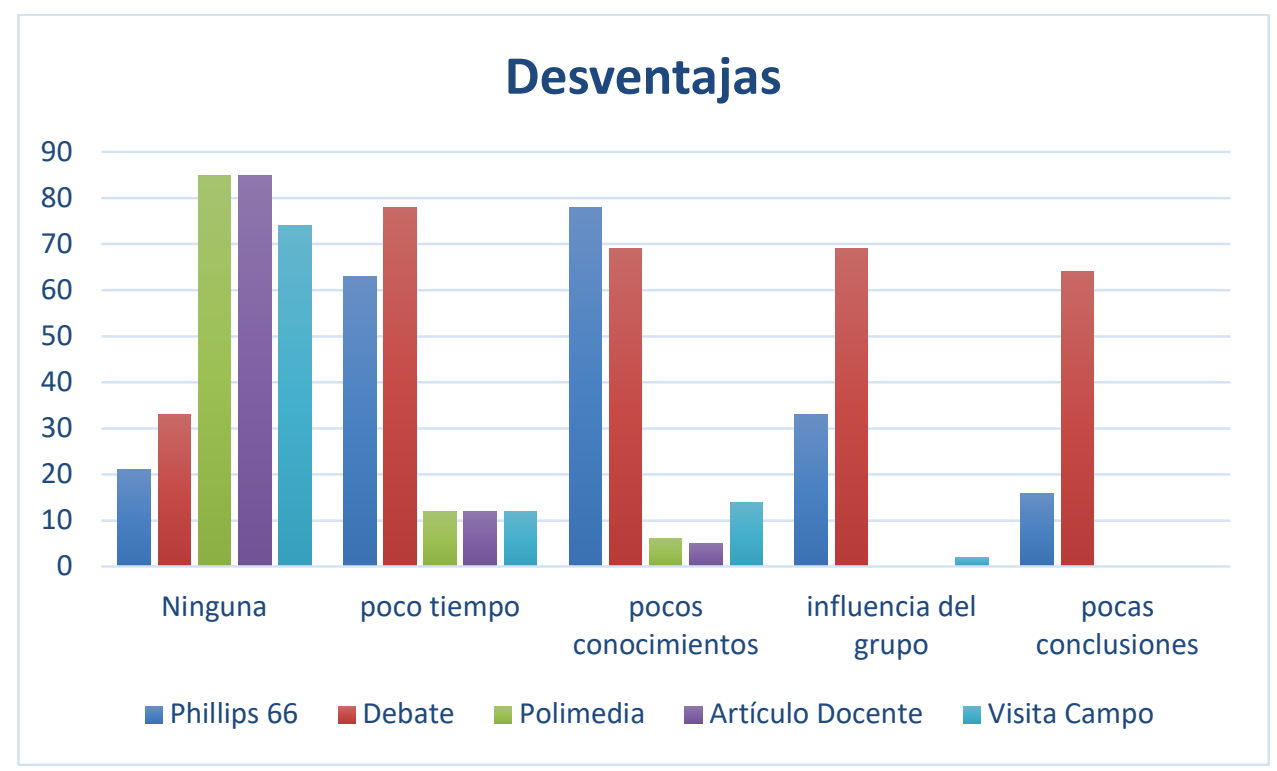

Fig. 6 Desventajas detectadas por parte del alumnado de cada actividad

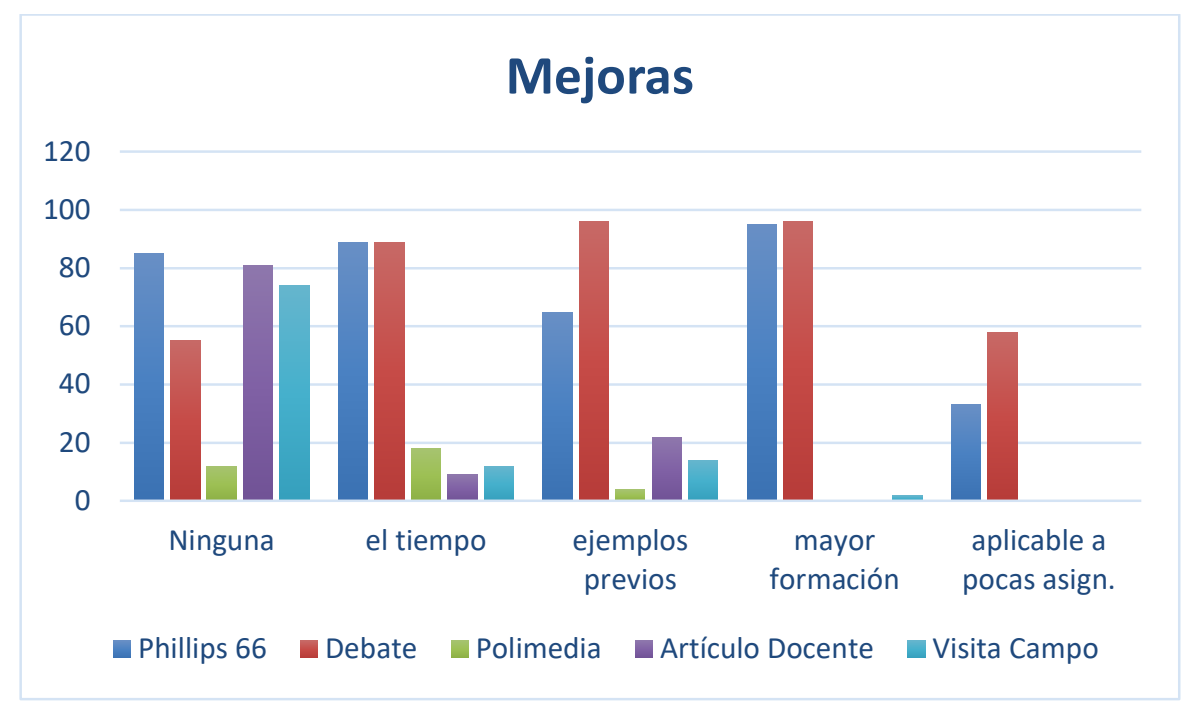

Fig. 7 Posibles mejoras a aportar por parte del alumnado

En el caso de la valoración, el sumatorio de todas las opciones sí es del 100\%. 


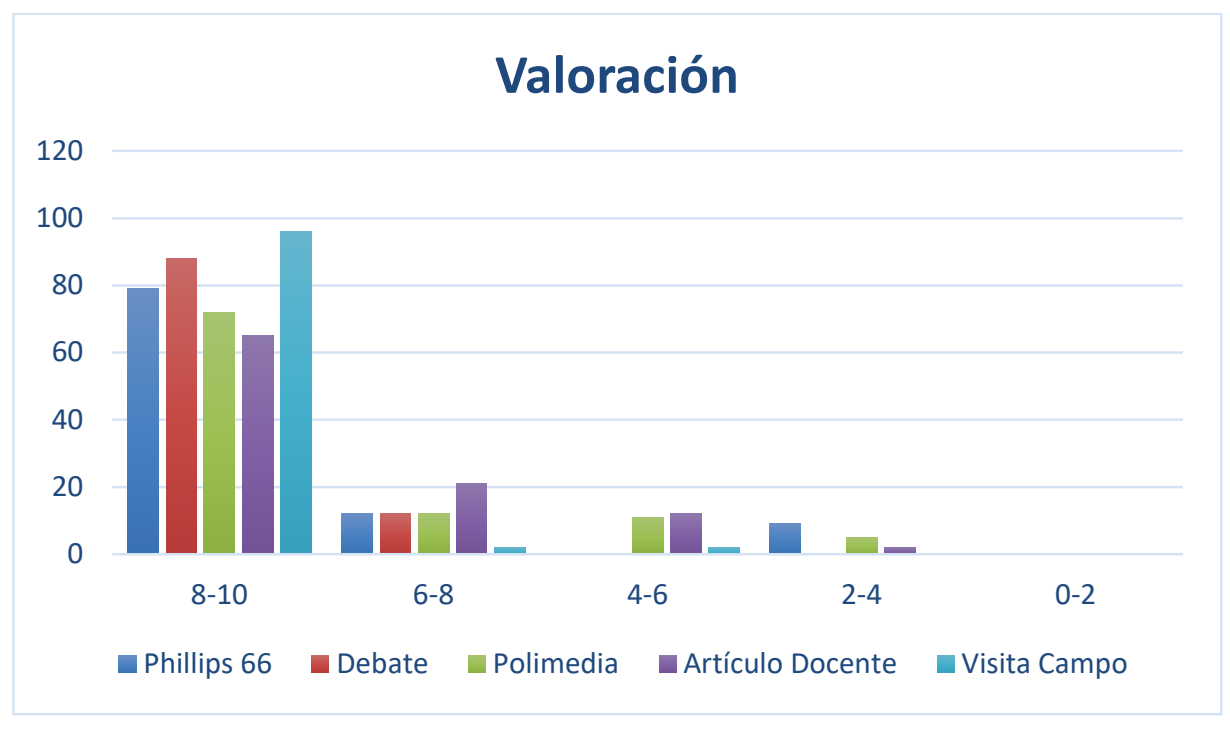

Fig. 8 Valoración general por parte del alumnado

A continuación, los autores resumen las principales conclusiones obtenidas tra el análisis de las actividades desarrollada:

- Método Phillips 66: La gran mayoría del alumnado admite no haber escuchado hablar nunca de dicho método. No obstante, en líneas generales el 91\% del alumnado lo considera un método dinámico, y con valores entre el 60 y el $72 \%$ del alumnado lo consideran entretenido, y útil para aprender nuevos conceptos y mejorar las dinámicas de grupo. En cuanto a las principales desventajas y posibles mejoras que los alumnos le proporcionan a este método es el tiempo dedicado a la actividad y los pocos conocimientos previos en la metodología de trabajo. En líneas generales el $79 \%$ del alumnado encuentra el proceso muy satisfactorio, como muestra su puntuación entre el 8 y el 10 en una escala del 1 al 10.

- Debate: pocos alumnos admiten haber participado previamente en debates sobre cuestiones técnicas. Al igual que en el método Phillips, el alumnado recomendaría una mayor formación sobre este tipo de actividades. En líneas generales lo consideran muy dinámico, entretenido y útil para mejorar las capacidades de exposición oral. En cambio consideran que se obtienen pocas conclusiones y que es aplicable a pocas asignaturas, lo que invita al profesorado a continuar mejorando esta metodología. En general más del $88 \%$ del alumnado lo considera muy satisfactorio.

- Polimedia: la herramienta de polimedias es mucho más conocida por parte del alumnado y ya poseen experiencia en su manejo. Admiten utilizarla sobre todo para el estudio previo a los exámenes. Solamente el 55\% lo considera un método dinámico, aunque el $78 \%$ considera que es fácil de aprender con vídeos polimedia. Por el contrario, y como era de esperar, esta metodología no potencia el trabajo en grupo ni las exposiciones orales. Los alumnos apenas aportarían mejoras a esta metodología siendo un $72 \%$ el alumnado que la considera muy satisfactoria. 
- Artículos Docentes: los artículos docentes se consideran poco dinámicos aunque faciliten el aprendizaje. Se trata de una metodología con mayor recorrido y mayor conocimiento por parte del alumnado. A pesar de que el $45 \%$ lo considera de poca utilidad, porcentajes por encima del $80 \%$ no aportaría mejoras, con lo que los autores consideran que se trata de una herramienta útil y con recorrido para el aprendizaje, pero mucho menos atractivo que vídeos polimedia o la creación de debates.

- Visitas de Campo: las visitas de campo se muestran indispensables para muchas asignaturas relacionadas con la ingeniería y con limitaciones de material en laboratorios o tecnología explicada. Los alumnos en su gran mayoría (cercana al 90\%) la consideran como una actividad entretenida y dinámica, no presentando desventajas salvo, como algunos alumnos comenta, la disponibilidad horaria. La satisfacción general es la más elevada (96\%), destacando además que los alumnos la consideran aplicable a la mayoría de asignaturas.

\section{Conclusiones}

En vista de los resultados obtenidos en las encuestas, las actividades de docencia inversa aplicadas al Grado de Ingeniería Química y al Master Universitario de Ingeniería, Procesado y Caracterización de Materiales, se han mostrado efectivas para la consolidación de aspectos teóricos, atractivas para el alumnado y con capacidad para sustituir total o parcialmente a la tradicional clase magistral. Si bien es cierto, que aunque los resultados muestran como todas las actividades se perciben atractivas y entretenidas, en técnicas como el Método Phillips 66 y los debates, el alumnado demanda de una mayor formación y tiempo sobre esta metodología.

Por otro lado, el estudio ha permitido al profesorado detectar los puntos fuertes y débiles de cada actividad, con el objetivos de mejorar los puntos débiles en futuros cursos académicos.

\section{Agradecimientos}

Este trabajo es fruto del esfuerzo que está realizando el Grupo de Innovación de Prácticas Académicas (GIPA) de la UPV en pro de la mejora docente. Los autores quieren mostrar su agradecimiento a la UPV y al Instituto de Ciencias de la Educación (ICE) de la UPV por su ayuda y colaboración en la formación del Equipo de Innovación y Calidad Educativa (EICE) denominado GIPA.

Así mismo los autores quieren agedecer al Vicerrectorado de Estudios, Calidad y Acreditación de la Universitat Politècnica de València la concesión del Proyecto de Innovacion y Mejora Educativa (PIME) referencia B18/18, dentro del cual se enmarca el presente trabajo.

\section{Referencias}

CRISPIN, M., L. y DORIA M. C. (2011). Aprendizaje autónomo orientaciones para la docencia. México: Universidad Iberoamericana. 
DALE, E. (1969). Audiovisual methods in teaching. New York: Rinehart and En: Winston.

ZABALZA, I., PEÑA, B., LLERA, E.M. y USÓN, S. (2016) "Improving the teachinglearning process using educational videos as reusable learning objects in the field of thermal engineering", En: Proceedings of the 8th International Conference on Education and New Learning Technologies (EDULEARN 2016), Barcelona: IATED Academy. 363-372 